\title{
HAITÍ: ¿LA MISIÓN DE LA ÚLTIMA OPORTUNIDAD?
}

\author{
Haiti: The Mission of Last Opportunity?
}

\author{
ROODY RESERVE \\ Pontificia Universidad Católica de Chile
}

\begin{abstract}
RESUMEN
En términos de los procesos sociales y políticos, 2013 terminó de la misma manera que lo hizo 2012: sin elecciones, protestas populares cotidianas y un enfrentamiento, a ratos incluso sangriento, entre partidarios y detractores de oposición y oficialista. El Diálogo Político entre haitianos, iniciado a mediados de enero de 2014 y auspiciado por los obispos católicos, intenta encontrar una solución al atasco político y hartazgo social. Este artículo hace un recuento de los principales acontecimientos sociales, económicos y políticos de 2013. Muestra que el voluntarismo desde la sociedad civil ha sido el camino elegido para desenmarañar los hilos del atascado proceso político. Subraya la novedad y muestra a la misma vez las limitaciones del llamado proceso de Diálogo Político Nacional.
\end{abstract}

Palabras clave: Diálogo Político Nacional, Senado, Ejecutivo, oposición, sociedad civil.

\begin{abstract}
In terms of its political and social processes 2013 ended the same way 2012 did. Elections were not held; there were massive and quotidian political protests, and street fighting took place between opponents and partisans of the president. The National Political Dialogue, held in the presence of the Episcopal Commision, tried to quell the violence and look for a solution to the social and political problems of the country. This article looks back at the political, social and economic events that took place during 2013. It shows that actors from the civil society have been very active in the search for a solution to the turmoil faced by Haiti during the year. They have sought, by means of voluntarism, to solve the problems of a stuck political process. The article shows both the merits and limits of the solutions they offered.
\end{abstract}

Key words: Nacional Political Dialogue, Senate, Executive, Opposition, Civil Society. 


\section{INTRODUCCIÓN}

La estabilidad ha sido uno de los elementos más esquivos en la larga travesía, hasta ahora hacia ninguna parte claramente discernible, que ha emprendido la sociedad haitiana desde 1986 (Jean-François, 2012). Allí están los datos de crecimiento económico en dientes de sierra para recordarlo. Lo mismo las periódicas sublevaciones sociales y catástrofes naturales. De igual modo, la larga lista de gobiernos provisionales, golpes de Estado, intervenciones extranjeras bajo la forma de misiones de paz, elecciones que no se han realizado a tiempo y otras inequívocamente fraudulentas recuerdan esta cruda realidad (Chérubin, 2013). En cierto modo, la realidad del país a lo largo de 2013 constituye un fiel reflejo de la persistencia del pasado. Un pasado que se expresa en forma de crisis permanente, en donde los cambios más importantes parecen de antemano condenados al fracaso, por el peso incalculable del pasado (Reserve, 2013).

Para conjurar el presagio de otro año frustrado, la Conferencia Episcopal de Haití lanzó la iniciativa de un diálogo político nacional entre los haitianos. Las líneas que siguen dan cuenta de este proceso de diálogo. Lo sitúan en un contexto más amplio de luchas enconadas entre los actores políticos y de debilidad institucional extrema que ha vivido el país en los últimos decenios. Este trabajo destaca las potencialidades del debate para el futuro político en Haití. Es la primera vez desde la transición que los actores más influyentes de la política nacional se sientan a dialogar bajo auspicio de la sociedad civil local. Esto abre una perspectiva interesante cuyo significado está aún por determinar. Sin embargo, se destaca al mismo tiempo los límites estructurales objetivos de un proceso cuya única baza descansa por ahora en la buena voluntad de sus protagonistas.

A nivel social el tema más importante ha sido el drama de los expulsados desde La República Dominicana. La decisión de La Corte Suprema de la República Dominicana decidió despojar de su nacionalidad a miles de dominicanos, en su mayoría de descendencia haitiana, so pretexto de la situación migratoria irregular de sus padres en el momento de su nacimiento. Esta decisión se dio además en un contexto de repatriación forzada de centenares de haitianos por parte de las autoridades dominicanas. En este trabajo ofrecemos una mirada panorámica de la realidad de algunos migrantes deportados, por medio de su reflexión sobre el trato que reciben tanto por parte de las autoridades de los dos países como de sus conciudadanos haitianos y dominicanos. La conclusión que se saca del relato de estos migrantes es que la mejora de su situación depende en gran medida de la capacidad del Estado haitiano para tomarse en serio y hacerse respetar por las autoridades de la república vecina.

El resto de este trabajo está estructurado de la siguiente manera. En la segunda sección se presentan de manera breve los principales indicadores económicos del 2013. Los esfuerzos de las autoridades por controlar las variables macroeconómicas estructurales han rendido frutos importantes. La inflación se ha mantenido bajo control y, a pesar de su insuficiencia, el crecimiento económico se ha mantenido. Sin embargo, el tema del empleo, la inversión internacional, siguen siendo una asignatura pendiente. La 
tercera sección alude al principal tema que ha afectado a los haitianos a lo largo del año recién pasado: las repatriaciones forzadas por parte de la República Dominicana. Estas repatriaciones, en muchos casos de personas que tienen poco vínculo con Haití, constituye por una parte un problema de derechos humanos que hay que atender incluso a nivel internacional, pero también a veces significa una presión para algunas comunidades que ven aumentar el número de repatriados. La cuarta sección versa sobre los esfuerzos que se han emprendido desde la sociedad civil, para superar el bloqueo político que ha afectado el país en el último lustro. Y, finalmente, en la conclusión se hace un llamado para ocuparse de un tema más teórico se trata de reflexionar sobre la pregunta de cuándo la "agencia", es decir la intervención de los actores, puede neutralizar el impacto de las estructuras.

\section{SITUACIÓN DE LA ECONOMÍA}

Según datos publicados por el Instituto Haitiano de Estadística e Informática (IHSI) la economía creció 4,3\% durante el 2013 (IHSI, 2013). ${ }^{1}$ Esta cifra quedó bastante lejos de la proyección de 6,5\% que hiciera el gobierno al principio del año (Le Nouvelliste, 2014). Aun así, puede leerse como un resultado positivo, que significa una mejora respecto de las cifras del año anterior en que el crecimiento fue tan solo de 2,9\% (Reserve, 2012: 227). Algo similar puede decirse de la inflación. Se ha observado una tendencia a la reducción. Este comportamiento concuerda con los objetivos de manejo sano de los parámetros macroeconómicos, definidos por el Banco Central en 2010 (CEPAL, 2013:3; BRH, 2008).

De estos datos de crecimiento económico, los responsables del gobierno haitiano destacaron principalmente la contribución de la agricultura. Es importante llamar la atención sobre este tema debido a las experiencias recientes de inseguridad alimentaria que han hecho noticias en Haití (Smith y Gélineau, 2012). Por ejemplo, durante 2010, las manifestaciones contra el hambre degeneraron en una crisis política importante que amenazó la estabilidad política del país. El hecho de que la rama alimentos haya crecido 10\% (CEPAL, 2013: 3), muy por encima del magro 4,3\% del PIB, indica que los esfuerzos del gobierno en este rubro están teniendo ciertos resultados. En esto, sin duda que también la naturaleza ha ayudado. Los huracanes y otros desastres naturales no afectaron con la virulencia de años anteriores. Así, se habrían salvado muchos cultivos que han permitido a los agricultores haitianos, en su gran mayoría personas que practican una agricultura de subsistencia y que no cuentan con seguros algunos que les proteja de los desastres naturales, producir suficientes granos básicos para nutrir a su familia y poner el excedente en el mercado local (CEPAL, 2013).

1 Esta cifra es ligeramente superior a la anunciada por la CEPAL para el mismo año. Pero aquí nos decantamos por la entregada por la institución haitiana. El documento de la CEPAl dice claramente que se trata de un estudio preliminar. Asumimos, entonces, que la discrepancia obedece a la naturaleza de las fuentes. En el caso del IHSI, un documento definitivo, y en el caso de la CEPAL no. 
Cuadro 1. Evolución de algunos indicadores macroeconómicos de Haití

\begin{tabular}{lrcr}
\hline & 2011 & 2012 & 2013 \\
\hline & & Tasa de variación anual & \\
\hline Producto Interno Bruto & 5,6 & $2,9^{\text {a }}$ & 4,3 \\
Producto Interno Bruto por habitante & 4,3 & 1,5 & 2,5 \\
Precios al consumidor & 8,3 & 7,6 & 4,2 \\
Dinero (M1) & 14,4 & 8,7 & 15,2 \\
Relación de precios del intercambio & $-17,0$ & 3,6 & 7,3 \\
\hline & & Porcentaje promedio anual & \\
\hline Resultado del gobierno central/PIB & 2,1 & $-0,8$ & $-0,8$ \\
Tasa de política monetaria & 3,2 & 3,0 & 3,0 \\
Tasa de interés activa nominal & 10,8 & 19,4 & 19,0 \\
\hline & & Millones de dólares & 1.077 \\
\hline Exportaciones de bienes y servicios & 1.017 & 1.046 & 3.807 \\
Importaciones de bienes y servicios & 4.154 & 3.841 & -287 \\
Balance de cuenta corriente & -339 & -359 & 0 \\
Balance de capital y financiera & 186 & 177 & \\
\hline
\end{tabular}

Fuente: Tomado de CEPAL (2013:1).

En la misma línea, según datos del gobierno, durante el 2103 más de novecientos mil haitianos habrían pasado de una situación de inseguridad alimentaria severa a una situación de inseguridad. Y doscientos cincuenta mil habrían salido completamente de la zona roja de la inseguridad alimentaria. Estos resultados, según el responsable de la Coordinación Nacional para Seguridad Alimentaria (CNSA), deben leerse como frutos directos del Programa de Apoyo a la Producción Agrícola Nacional impulsada por el gobierno del presidente Martelly. ${ }^{2}$

Sin embargo, importantes actores de la sociedad civil, que trabajan con amplios sectores del campesinado, junto a pequeños productores y personas en situación de inseguridad alimentaria, contestan la veracidad de las cifras gubernamentales. Al contrario, critican la decisión gubernamental de promover el acaparamiento de parcelas agrícolas por parte de grandes compañías extranjeras, en detrimento de los pequeños productores.

2 Esta información fue dada a conocer durante la celebración de un encuentro televisado del gobierno. En efecto, desde hace algún tiempo, el primer ministro haitiano Laurent Salvador Lamothe ha instaurado la costumbre de filmar los encuentros de su gobierno para que toda la población pueda acceder a los contenidos de discusión del gabinete. Este vigésimo tercer encuentro gubernamental estaba convocado precisamente bajo el lema de relanzar la Agricultura y la Seguridad Alimentaria. Ver: http://www.communication.gouv.ht/archives/2769 [Consultado el 15 de enero de 2014]. 


\section{COYUNTURA SOCIAL}

A lo largo del año recién pasado el gobierno ha mostrado una mayor tendencia a gastos sociales. Motivado en parte por el proceso electoral en ciernes, por otra parte por las manifestaciones que se organizan a diario para pedir la dimisión del Presidente, la inversión social se ha usado en cierta medida para apaciguar los ánimos, comprar algunas voluntades y aportar cierto alivio a buena parte de la población, que sin esta ayuda difícilmente habría podido subvenir a necesidades tan básicas como la alimentación de sus familias. Aun cuando no se cuenta con datos que cubran todo el año en materia de gastos sociales, cabe destacar el crecimiento que ha tenido este rubro en los primeros tres meses de 2013, en comparación con 2012. El cuadro siguiente muestra el comportamiento de este rubro durante seis meses, entre 2012 y 2013. Estas cifras indican claramente que el gobierno tuvo motivos importantes para hacer notables inversiones sociales durante los primeros meses del año. Estos meses, cabe recordar, eran particularmente calientes, en términos de manifestaciones sociales y políticas.

Por otro lado, según muchos observadores, el gobierno ha aumentado su capacidad de intervención con los programas sociales como Ti manman Chérie, Restaurantes Comunitarios y apoyos monetarios a estudiantes universitarios. No se cuenta con cifras oficiales de cuánto ha aumentado la cobertura de dichos programas, comparando con las cifras de 2012, por ejemplo (Reserve, 2013). Sin embargo, expertos locales y oficiales del gobierno consideran que estos programas tienen una cobertura muy importante, especialmente en las zonas más desfavorecidas donde se han usado para intentar atajar la manipulación de ciertos habitantes por parte de opositores políticos para generar protestas populares.

Cuadro 2. Gastos sociales en millones de gourdes con fondos provenientes del Presupuesto General de la República

\begin{tabular}{lrrrrrr}
\hline & Oct. 2012 Nov. 2012 Dic. 2012 & Ene. 2013 & Feb. 2013 Mar. 2013 \\
\hline Min. de Agricultura & 2,1 & 87,7 & 105,1 & 332,8 & 207,1 & 84,3 \\
Tasa Ejecución & 0,1 & 4,2 & 9,1 & 24,5 & 34,2 & 38,1 \\
Tasa Crecimiento (porcentaje) & $-92,6$ & 158,8 & 54,7 & 880,7 & 297,2 & $-65,1$ \\
Ministerio de Educación Nacional & 12,2 & 735,6 & 838,8 & $2.483,7$ & 425,7 & 440,3 \\
Tasa de Ejecución & 0,1 & 8,9 & 18,8 & 48,3 & 53,4 & 58,6 \\
Tasa de crecimiento (porcentaje) & $-88,9$ & 2,8 & $-20,2$ & 428,5 & 19,8 & $-6,9$ \\
Ministerio de Salud Pública & 10,7 & 316,3 & 317,4 & 815,8 & 251,4 & 250,1 \\
Tasa de Ejecución & 0,3 & 10,5 & 20,6 & 46,8 & 54,8 & 62,8 \\
Tasa de crecimiento (porcentaje) & $-91,9$ & 131,4 & 17,5 & 437,7 & 53,1 & 42,2 \\
Total (en millones de gourdes) & 25 & 1.140 & 1.261 & 3.632 & 884 & 775 \\
Tasa de Ejecución & 0,2 & 8,5 & 17,7 & 44,2 & 50,7 & 56,3 \\
Tasa de crecimiento (en porcentaje) & $-90,7$ & 28,5 & $-9,2$ & 454,1 & 54,7 & $-12,9$ \\
\hline
\end{tabular}

Fuentes: tomada de la página web del Ministerio de Finanzas. 
A falta de grandes huracanes destructores, un tema que ha marcado la vida social haitiana a lo largo del año recién pasado ha sido el de la deportación masiva de haitianos por parte de las autoridades de la República Dominicana y la decisión de la corte Suprema de ese país de despojar de su nacionalidad a los hijos de los inmigrantes, en su mayoría de descendencia haitiana, que se encuentren desde 1929 en este país. A junio de 2013, el sitio web de Información y Análisis de América Latina había contabilizado ya 3.434 casos de expulsión en condiciones inhumanas de haitianos residentes en el país vecino. Como indican las noticias, no solo se expulsan a personas indocumentadas, sino también muchas veces a ciudadanos dominicanos por tener tonos de piel que los funcionarios dominicanos identifican como haitianos. Estas expulsiones siguieron a lo largo de todo el año y aumentaron considerablemente entre junio y diciembre de 2013. A este respecto las noticias subrayaban que "entre los expulsados se encontraban tres personas de Sri Lanka, las cuales fueron dejadas con el resto del grupo [de haitianos] en la puerta fronteriza de Comendador-Belladere, a más de 15.000 kilómetros de su casa". ${ }^{3}$

El aumento de las expulsiones masivas de haitianos en República Dominicana que se experimentó en 2013 se dio en un contexto más amplio de un fallo de la Corte Suprema de Justicia de ese país en que despojó de la nacionalidad dominicana a los hijos de extranjeros que se habrían encontrado en situación irregular en su país desde el inicio del siglo XX. Esa decisión ha generado el repudio internacional, de muchas organizaciones y gobiernos que han denunciado su carácter racista y su orientación especial hacia los descendientes de emigrantes haitianos que llegaron a trabajar en el sector azucarero, de la construcción últimamente, en la república vecina. Países miembros del Mercado Común Caribeño (CARICOM) y la misma organización han condenado sin ambages la decisión de las autoridades judiciales dominicanas.

En este marco, la Comisión Interamericana de Derechos Humanos (CIDH) desarrolló una visita a República Dominicana, entre el 2 y el 5 de diciembre de 2013, para observar la situación del goce del derecho a la nacionalidad, la igualdad, la identidad y la no discriminación. Sus conclusiones preliminares que se reproduce en extenso aquí dice lo siguiente:

"La Comisión estima que la sentencia del Tribunal constitucional conlleva a una privación arbitraria de la nacionalidad. La sentencia tiene un efecto discriminatorio, dado que impacta principalmente a personas dominicanas de ascendencia haitiana, quienes son personas afrodescendientes; priva de la nacionalidad retroactivamente; y genera apátrida respecto de aquellas personas que no son consideradas como nacional suyo por ningún Estado, conforme a su legislación. (...). Muchas de las personas afectadas por la sentencia 168/13 del Tribunal Constitucional son personas que nacieron en República Dominicana y que anteriormente han sido reconocidas por el Estado como dominicanas, a través de la expedición de un documento por parte de las instituciones estatales certificándolos como tal. Estas personas 
tienen estrechos lazos en República Dominicana: han pagado impuestos y generado riqueza, han contribuido a la seguridad social, se criaron y educaron en el país, y tienen establecido allí su núcleo familiar. En sus testimonios ante la CIDH expresaron tener un profundo sentimiento de identidad dominicana. Como lo expresó un joven en Santo Domingo: "yo nací bajo la bandera dominicana y no conozco ninguna otra bandera".

Estas personas están afectadas por decisiones arbitrarias adoptadas por la Junta Central Electoral a lo largo de los últimos años. La Comisión recibió información de que numerosos funcionarios de esta dependencia niegan la documentación a las personas nacidas en República Dominicana. Inclusive, en algunos casos, después que tribunales ordenaran que dichos documentos fueran expedidos, la Junta Central Electoral desacató la orden judicial. Algunos servidores de esta dependencia privan a las personas de su nacionalidad, en forma arbitraria y discrecional.

La falta de reconocimiento de la personalidad jurídica de estas personas como resultado de que no están registrados o de las dificultades en el acceso a la cédula genera una situación de extrema vulnerabilidad en la cual se producen violaciones a otros derechos humanos, en un círculo vicioso que solo se puede romper a través del reconocimiento de su nacionalidad.

La negación de los documentos por parte de la Junta Central Electoral crea obstáculos a las personas en el ejercicio de su derecho a la libre circulación en el país, ya que se quedan sin prueba de su nacionalidad dominicana. Según un testimonio recibido por la CIDH, a una mujer de ascendencia haitiana le fueron requeridos documentos que probaran su nacionalidad dominicana por parte del conductor de un autobús de transporte público. Otra mujer dijo que por miedo a ser deportada a Haití, "donde yo no tengo a nadie", ella no viaja a ver a sus nietos, que viven en una ciudad ubicada a menos de una hora de donde ella vive.

La denegación de documentos a un gran número de personas nacidas en República Dominicana fue una práctica extendida a lo largo de los últimos años, cuando también se estaban registrando deportaciones y expulsiones arbitrarias. En esas deportaciones estuvieron incluidas personas nacidas en República Dominicana, a quienes el Estado dominicano les había reconocido su nacionalidad dominicana a través de la expedición de actas de nacimiento y cédulas de identidad. La Comisión Interamericana valora como positiva la orden del Presidente de la República de suspender las deportaciones. No obstante, la Comisión recibió información en terreno de que las deportaciones continúan, aunque a un ritmo menor que el de años anteriores.

La Comisión Interamericana observó asimismo que la desnacionalización afecta principalmente a personas de ascendencia haitiana nacidas en República Dominicana. La sentencia 168/13 del Tribunal Constitucional tiene un efecto desproporcionado sobre estas personas, en virtud de que constituyen la mayor parte de la población migrante del país. La Corte Interamericana ha establecido que existe un vínculo indisoluble entre la obligación de respetar y garantizar los derechos humanos y el principio de igualdad ante la ley y no discriminación, y que este debe impregnar toda la actuación del Estado. En ese sentido, el Estado no puede actuar contra un determinado grupo de personas, por motivos como raza, etnicidad, u origen nacional, entre otros". 
Fuerza es de constatar, no sin cierta ironía, que al mismo tiempo que las autoridades dominicanas se han empeñado en discriminar a sus connacionales de ascendencia haitiana o simplemente de piel oscura, los negocios de grandes empresarios dominicanos en Haití han florecido en los últimos años. Como una muestra de ello, según las cifras publicadas de empresas contratadas para la construcción de caminos y otras grandes obras de infraestructura en ejecución, una empresa dominicana denominada Estrella, ligada a un influyente senador dominicano, Félix Bautista, ha obtenido en los últimos tres años cerca del $25 \%$ de los contratos, en el marco de la reconstrucción posterremoto. Esto equivale a una cifra cercana a unos ciento cincuenta millones de dólares americanos.

Esta realidad no deja indiferentes a los deportados, víctimas en carne propia de las arbitrariedades y racismo de las autoridades dominicanas. En una visita de investigación entre el 5 y 14 de febrero de 2014 en Fonds Parisien, una localidad haitiana fronteriza donde trabaja el Servicio Jesuita para Migrantes en asentamientos de retornados, algunos deportados reflexionaban acerca de la indiferencia de las elites políticas haitianas frente a los problemas que sufren en República Dominicana. Para muchos la debilidad del Estado haitiano $0^{4}$ y la insensibilidad de sus autoridades, explican en buena medida las arbitrariedades de las que son víctimas en territorio dominicano.

Yolette, una deportada de 22 años, que llegó con su madre a República Dominicana cuando tenía 6 años, ahora madre de 2 niñas de 7 y 5 años nacidas en República Dominicana y que fue expulsada a principios de 2013, lo puso en estos términos: "Las autoridades dominicanas nos miran y tratan como animales porque saben que autoridad haitiana alguna les va a pedir cuenta o denunciar sus violaciones en contra de nosotros" (entrevista personal, 5 de febrero de 2014).

Jean, un señor de 70 años, que vivió durante más de 40 años como cortador de caña en un batey de la provincia de Higuey, y que retornó hace unos cinco años por su propia cuenta, sostiene que "es una vergüenza que las autoridades haitianas sigan contratando a firmas dominicanas para hacer carreteras y otras obras de construcción en Haití mientras el gobierno dominicano sigue tratando como perros a los haitianos". A su juicio, "tiene que haber soborno de por medio. Porque, de lo contrario, no es posible que un gobierno pueda ser tan insensible al sufrimiento de sus propios connacionales" (entrevista personal, 5 de febrero de 2014).

Otro tema importante de destacar es la particular situación social de los deportados. Devueltos, en su mayoría, a un país que conocen apenas, constituyen uno de los grupos más vulnerables de la sociedad haitiana. Esto es particularmente cierto en los casos de los deportados que se quedan en las zonas fronterizas, en vez de retornar a las comunidades de donde son originarios. Viven hacinados en comunidades que no cuentan con los servicios básicos. Además, tal como manifestaron algunos, al no contar siquiera con

4 Ver Corten, 2011, para una reflexión comparativa sobre los Estados haitiano y dominicano. Allí, el autor destaca las debilidades de ambos Estados, especialmente el haitiano que, partiendo de una condición similar con la República Dominicana, ha sufrido una erosión constante en su capacidad de satisfacer las necesidades básicas de su población. Sin duda que las condiciones materiales de los haitianos en Haití constituyen por una parte importante la explicación del origen de la migración al país vecino. 
documentos de identidad haitiana, ni siquiera son atractivos para los candidatos que pasan a regalar cosas o comprar votos durante las campañas electorales.

Therese, una retornada de 65 años, que dejó casa y familia en República Dominicana, contó lo siguiente: "Yo me beneficiaba de la política en República Dominicana. Durante las campañas electorales pasaban los políticos del PLD al barrio, anotaban el número de las cédulas y entregaban víveres de los cuales yo también era beneficiada. Pero ahora en Haití los políticos ni siquiera llegan a este barrio. Los candidatos a diputados y a la Municipalidad, al saber que no contamos con documentos, se van a otros barrios de gente de por acá, que pueden comprometer sus votos para el día de las elecciones" (entrevista personal, 5 de febrero de 2014).

\section{COYUNTURA POLÍTICA}

Las manifestaciones diarias marcaron de nueva cuenta la cotidianeidad política haitiana a lo largo de 2013. Frente a la incapacidad de los actores políticos para ponerse de acuerdo sobre un calendario para realizar las elecciones, la solución que encontraron los opositores al gobierno de Martelly fue pedir su expulsión de la presidencia del país ${ }^{5}$. En este sentido casi a diario se podían encontrar en la prensa reportes de manifestaciones, de calles bloqueadas y enfrentamientos violentos, a veces incluso con el uso de armas de fuego, entre manifestantes y policías por un lado, y opositores y adeptos del gobierno por otro. Desde esta perspectiva, el año ha sido agitado políticamente.

De modo que 2013 se inició con una sobrecarga política en donde la fricción y el atasco predominaban. Si bien se resolvió el problema de la composición del organismo electoral que debería de organizar estas elecciones intermedias, los actores opositores no quedaron conformes con ciertos personajes, cercanos al ejecutivo, que conformaban dicho organismo. De modo que la sensación generalizada era que el país había entrado en un camino sin retorno en que la solución definitiva tal vez iba a ser de nueva cuenta la dimisión del Presidente. ${ }^{6}$ Para atajar esta salida, incluso actores internacionales claves como los

5 El pasado 25 de septiembre de 2013 el Senado aprobó que se abriera un antejuicio contra el Presidente de la República por supuestamente estar implicado en la muerte del juez Jean Serge Joseph. Ver: http://rapadoo. com/2013/09/25/le-matin-le-senat-approuve-la-demande-de-la-mise-en-accusation-du-president-martelly/ [Consultado, 12 de enero de 2014]. El juez murió repentinamente, según las informaciones recogidas por una comisión de investigación del Senado, luego de haber sido convocado por el Presidente y el primer ministro para que se explicara por la decisión de convocar a la familia del Presidente, su esposa y un hijo, por malversación de fondos públicos, luego de una demanda interpuesta en contra de ellos por unos abogados. Supuestamente las presiones ejercidas contra el juez fueron tan intensas que este falleció por ello. http://www. alterpresse.org/spip.php?article15176\#.UzEAKqh5NQ8 [Consultado el 12 de enero de 2014]. En el momento en que se escriben estas líneas, la Cámara de Diputados, en la que el Presidente cuenta con el respaldo de la mayoría de sus miembros, no había decidido aún lo que iba a hacer de dicha decisión de los senadores. Según la Constitución haitiana, incumbe a los diputados erigirse en corte de justicia en caso de alguna acción judicial en contra del Presidente.

6 Los antecedentes recientes se encuentran en las dos salidas prematuras del gobierno del expresidente Jean Bertrand Aristide, especialmente en 2004, cuando lo derrocó una coalición de grupos armados. 
embajadores de los Estados Unidos y de Francia en Haití, han tenido que manifestar públicamente su respaldo al Presidente, que consideran debe de terminar su mandato.

Fue en estas condiciones estructurales que la Conferencia de obispos católicos haitianos lanzó la iniciativa del diálogo entre haitianos. ${ }^{7}$ Esta iniciativa, que se dio a conocer a principios de 2014, se inició en realidad a finales de 2013, con reuniones discretas e informales de contacto con todos los actores políticos importantes, según lo que nos comentó Jean-Marie Louis, Decano de la Facultad de Humanidades de la Universidad Notre Dame de Haití, uno de los asesores del presidente de la Conferencia Episcopal y recién nombrado como el primer cardenal de la Iglesia católica haitiana, Mgr Chibly Langlois.

Según Jean-Marie Louis, primero lo que se busca con este diálogo es ofrecer un respiro a la sociedad haitiana frente a un impasse político que amenaza con desestabilizar la sociedad. En segundo lugar, se espera poner las bases para generar confianza entre los actores, una cultura de diálogo y de negociación para poder abordar temas más sustanciales en el futuro, en torno a la construcción de un gran acuerdo nacional de gobernabilidad y de desarrollo económico y social.

Efectivamente, entre el 24 de enero y 13 de febrero se reunieron en el hotel "El Rancho" de la capital haitiana, representantes de los poderes ejecutivo y legislativo y miembros de partidos políticos nacionales para iniciar un diálogo abierto para enfrentar los principales problemas políticos que mantenían estancado el país desde 2011. Se llegó a un acuerdo para organizar elecciones para las 2/3 partes del Senado, y las colectividades territoriales; se acordó instaurar un consejo electoral provisional para reemplazar el Colegio Electoral Transitorio del Consejo Electoral Permanente que se había nombrado el año anterior (Reserve, 2013); y se acordó nombrar un gobierno de apertura, que permitiría ampliar el gobierno actual a la participación de otros partidos de oposición.

Sin embargo, en el momento en que se escribe este trabajo el acuerdo no ha sido firmado aún. La ceremonia de firma prevista para el 14 de febrero fue abortada, debido a la negativa del presidente del Senado quien había puesto como condición que el Presidente firmara y publicara el decreto de nombramiento de los nueve miembros de la Cour Superieure des Comptes (la Corte de Cuentas). En efecto, hace más de cinco meses, tal como está prescrito en la Constitución de la República, el Senado designó a las nueve personas que deberían integrar esta corte que tiene como misión supervisar el manejo de las cuentas del país. A pesar de que la carta magna da diez días al Presidente para emitir reservas sobre las personas designadas, este nunca presentó objeciones y tampoco publicó en el diario oficial el nombre de los jueces escogidos por el legislativo. De modo que cinco meses después el presidente del Senado puso como condición para rubricar el acuerdo la publicación oficial de la lista de los jueces designados.

Para salvar parcialmente la situación, la víspera de la fecha de la firma de los protagonistas para cerrar el diálogo, el Presidente de la República decidió publicar el nombre de seis

Participan directamente en el diálogo, el Senado de la república, especialmente su presidente, cuya mayoría es hostil al Presidente de la República y votaron para pedir a la Asamblea Legislativa su enjuiciamiento. También están los partidos políticos, de oposición y los afines al gobierno del presidente Joseph Michel Martelly. 
personas y objetar los documentos profesionales de otros tres magistrados, bajo el pretexto de encontrar alteraciones fraudulentas en sus títulos universitarios. Mientras tanto, los líderes del partido más influyente en la vida política haitiana en los últimos veinte años decidieron abandonar el diálogo. ${ }^{8}$ De modo que la firma del acuerdo quedó en suspenso mientras el propio mediador y los actores políticos viajaban a Roma para participar de la ceremonia de creación del cardenal Langlois. Se espera que a su regreso y luego de la celebración del carnaval se buscaría una solución al punto controversial que impide la conclusión exitosa del diálogo.

Todavía está temprano para dimensionar los impactos reales de este primer ejercicio de diálogo entre haitianos, bajo el auspicio de la sociedad civil local. Aun así, muchos actores han destacado su importancia. Es la primera vez desde la transición que los haitianos dialogan entre ellos, con la mediación de una autoridad moral interna. Diálogos anteriores, especialmente las largas negociaciones relacionadas a temas de elección o el mismo retorno de Aristide al poder en 1995, luego del golpe de Estado de 1992, se dieron bajo el auspicio de potencias externas que tenían la capacidad de presionar a uno y otro e imponer las concesiones a cada uno de los participantes en el diálogo.

La experiencia de este último diálogo muestra que la Iglesia católica haitiana está retomando ciertos protagonismos y legitimidad que había perdido luego del fin de la dictadura, por el involucramiento directo de sus líderes en las crisis políticas, como parte de los bandos en conflicto. Desde una perspectiva instrumental de la sociedad civil (Huntington, 1968, 1991; Inglehart, 1992), no se puede más que celebrar esta legitimidad recobrada de un actor clave de la sociedad civil.

Ello no obsta, sin embargo, para plantearse la pregunta de hasta qué punto la apuesta voluntarista será suficiente para superar las taras estructurales que hacen que la desconfianza, la apuesta compulsiva por el corto plazo, siga predominando entre los actores políticos nacionales. El caso de la traba para la firma de los acuerdos, y las exigencias posteriores de otros senadores -exigencias adicionales a la publicación del nombre de los miembros restantes de la Corte de Cuentas-, muestra que la lógica de la desconfianza sigue imperando.

Dicho de otra manera, cabe preguntarse si la personalidad y el voluntarismo de los actores políticos y de los miembros de la sociedad civil será suficiente para vencer la resistencia de las estructuras. Esta pregunta plantea una discusión más amplia, la de cómo escapar de la estructuras, si asumimos que estas condicionan en demasía los resultados que se puedan esperar de la cooperación entre los actores políticos. En Haití, tal como muchos autores lo han reseñado, desde el inicio de la transición, en 1986, se ha impuesto la idea de que es más rentable apostar por la derrota definitiva del adversario, en vez de cooperar y ceder algunas cuotas de poder en un proceso de negociación limpio.

La tozuda realidad ha mostrado que en la medida en que perseveren los extremistas en materia política, a pesar de los costos enormes que ello supone para la población

8 La representante del partido Fanmi Lavalas, del expresidente Aristide y la representante del partido Fusión decidieron abandonar las discusiones antes de alcanzar la etapa de firma del documento. 
siempre han terminado consiguiendo sus objetivos. Primero fueron los militares que apostaron por descarrilar el proceso de transición democrática. A continuación, Jean Bertrand Aristide les ganó la partida, disolviendo la institución castrense cuando pudo retornar protegido por las armas del Ejército de los Estados Unidos. Luego los enemigos de Aristide volvieron a derrotarlo, mandándolo al exilio a Sudáfrica. Así, sucesivamente, las apuestas extremas han terminado ganando la partida. Por lo que no es descabellado que aquellos que hoy se movilicen para conseguir la salida del poder de Martelly, tengan cierta convicción de lograr sus cometidos. Al menos la historia reciente ha mostrado que es factible conseguir tales objetivos. Además, ¿qué ganan con negociar, si históricamente quienes lo han hecho de buena fe han sido traicionados luego por sus contrapartes?

En estos escenarios, la literatura nos dice que solo una coyuntura crítica (Collier y Collier, 1991; Mahoney 2001; Yashar, 1999) puede obligar a los actores a cambiar el curso del camino que ellos han seguido casi ciegamente. Desde esta perspectiva, cabe el pesimismo respecto de los resultados del proceso de diálogo que han emprendido los actores. Ello, porque nada ha cambiado estructuralmente como para esperar un "arrepentimiento" de los líderes políticos. Además, ni siquiera ha habido un atisbo de renovación en los actores principales. La mayoría de quienes estaban alrededor de la mesa de negociación son los mismos que hace un decenio se habían enfrentado a muerte para el control de las instituciones políticas y económicas.

Ante este escenario, tal vez solo la lucidez de la reminiscencia puede catapultar hacia un comportamiento de respeto y de cooperación entre los políticos haitianos. En este sentido, solo una fe, casi ciega en la importancia del factor humano puede hacer esperar un desenlace distinto a lo que por lógica y experiencia cabría esperar del proceso de diálogo entre haitianos. Quizá solo porque le mediador sea una figura religiosa cabe esperar contra toda esperanza que algo duradero salga de las discusiones para encarrilar el tren político.

\section{CONCLUSIONES}

En este trabajo hemos presentado una reseña de los principales acontecimientos sociales, económicos y políticos de Haití a lo largo de 2013. Por un lado destacamos el comportamiento regular de la economía. No obstante el bajo crecimiento, debido a las necesidades de la economía y sociedad haitiana, es importante recalcar este resultado positivo, en un contexto de marasmo político.

Desde el punto de vista social, el gobierno ha consentido importantes gastos sociales, financiados en su mayoría por los fondos provenientes del Petro Caribe que concede importantes préstamos al gobierno haitiano para invertir en programas de construcción de infraestructuras, a tasas muy bajas y a un plazo bastante lejano. Amparado en ello, el gobierno de Martelly ha podido dar la sensación de mantener el país en construcción. De hecho, al arribar a la capital haitiana la sensación que tiene el visitante es que se trata de un país en construcción. Porque prácticamente en cada calle hay un espacio cerrado o de autopistas en construcción o de edificios gubernamentales por construir. 
A nivel social, la agricultura ha sido el principal motor del crecimiento económico observado. Junto con las remesas que envían los haitianos residentes en el exterior, han sido los sectores más dinámicos de la economía. Si bien los huracanes y desastres naturales no se hicieron sentir a lo largo de 2013, la deportación constante y masiva de haitianos por parte de la República Dominicana ha tenido un impacto importante en la dinámica social. Como hemos visto, las personas que retornan de esta manera, dejando todas sus pertenencias en el país vecino, se encuentran entre los más precarios de la sociedad.

Finalmente a nivel político, la iniciativa de diálogo entre haitianos, auspiciada por la Conferencia Episcopal de la Iglesia católica haitiana, ha ofrecido cierto respiro al atasco político que había prevalecido en los últimos tres años. Sin embargo, no obstante las ambiciones y anhelos de la sociedad civil, hemos argumentado que es difícil esperar un cambio sustancial en el comportamiento de los actores políticos, por el peso de la tradición y la memoria de las traiciones pasadas.

En resumidas cuentas, la esperanza para un mejor 2014 en Haití pasa por la voluntad política de los actores. La única apuesta es que, ya que se trata en su mayoría de los mismos actores que han dominado la política en los últimos veinte años, hayan aprendido, por haberlo repetido hasta la saciedad, que la no cooperación lleva al suicidio colectivo. Solo de esta manera se pueda esperar un cambio de rumbo, sin que se haya producido una coyuntura crítica que se los obligue.

\section{REFERENCIAS}

Cherubin, G. 2013. Le ventre pourri de la bête. Port-au-Prince: Editions de l’Université d’Etat d'Haiti.

Corten, A. 2011. L'État faible, Haiti et la République Dominicaine. Montréal: Mémoire d'Encrier.

Jean-Francois, H. 2012. Haiti autrement. Port-au-Prince: Editions Mediatek.

Reserve, R. 2013. "Haití, cuando el pasado es demasiado pesado". Revista de Ciencia Política 32 (2): 225-245.

Smith, Amy Erica, y François Gélineau. 2012. The Political Culture of Democracy in Haiti and in the Americas, 2012: Towards Equality of Opportunity. Nashville: Latin American Public Opinion Project.

Roody Reserve es Candidato a doctor en Ciencia Política para la Pontificia Universidad Católica de Chile. Actualmente trabaja como docente en la Universidad Centroamericana José Siméon Cañas, en el Departamento de Sociología y Ciencia Política. rreserve@uca.edu.cl 
